\title{
Synthesis and Characterization of Butyryl Cyclosophoraose, and its Inclusion Complexation Behavior for Some Flavonoids
}

\author{
Yongeun Kwon, Eunae Cho, Im-Soon Lee, ${ }^{\dagger}$ and Seunho Jung ${ }^{*}$ \\ Department of Bioscience and Biotechnology, Bio/Molecular Informatics Center, Center for Biotechnology Research at UBITA \\ (CBRU), Konkuk University, Seoul 143-701, Korea. *E-mail: shjung@konkuk.ac.kr \\ †Department of Biological Sciences, Center for Biotechnology Research at UBITA (CBRU), Konkuk University, Seoul 143-701, Korea \\ Received June 7, 2011, Accepted June 14, 2011
}

Key Words : Butyryl cyclosophoraoses (butyryl Cys), Cyclosophoraoses (Cys), Inclusion complex, Flavonoids, Solubility

Cyclosophoraoses (Cys) are unique molecules that are found in Agrobacterium and Shinorhizobium species. ${ }^{1}$ They play an important role in osmoregulation and are involved in the initial stage of root nodule formation during nitrogen fixation. $^{2}$ They are $\beta-1,2$ linked cyclic oligosaccharides, which are produced as a mixture of large ring molecules ranging from 17 to 23 in their degree of polymerization (DP). Although their exact three-dimensional structures are not clearly known, the conformational analyses indicate that Cys seem to have flexible backbone and narrow cavity sizes. ${ }^{3}$ Based on the structure, the ability to form inclusion complex with guest molecules has been studied as a solubility enhancer of poorly soluble guests or a chiral selector. $^{4,5}$

For further application of Cys, it is possible to modify the hydroxyl group with another functional group. A recent study has reported some modified Cys such as carboxymethylated Cys and sulfated Cys, as a solubility enhancer of $\mathrm{N}$-acetylphenylalanine and a chiral selector of some flavonoids. ${ }^{6,7}$ The result indicates that substituents (carboxymeth$\mathrm{yl}$ and sulfate groups) in the modified Cys contribute to the complexation ability different from native Cys. In this way, the complexation ability of modified Cys can be expanded and differentiated by the substituent characteristics. Herein, we synthesized butyryl cyclosophoraoses (butyryl Cys) as a novel host molecule, which are endowed with hydrophobic side chain. Native Cys were modified with acyl group which was butyryl chloride through chemical derivatization, and their modified structures were investigated with two analytical methods, nuclear magnetic resonance (NMR) spectroscopy and matrix assisted laser desorption/ionization-time of flight (MALDI-TOF) mass spectrometry. Finally, the butyryl Cys were investigated for inclusion complex formation with some flavonoids.

Flavonoids, polyphenolic phytochemicals, are plant secondary metabolites found in vegetables and fruits. They are classified as four subgroups (flavones, flavanone, flavonol, and isoflavone) based on the backbone structure. Although they have many physiological effects as antioxidants and anticarcinogens, the potential clinical utility is limited because of the low aqueous solubility. ${ }^{8}$ For this reason, there has been growing interest in the improvement of flavonoid solubility by inclusion complexation. ${ }^{9}$ As a novel complexation agent for flavonoids, butyryl Cys were evaluated in the present study.

The butyryl Cys was synthesized through one-step reaction (Scheme 1)..$^{10}$ The reaction was monitored on TLC, and the $\Delta R_{f}$ value of native Cys and butyryl Cys was $0.33 .^{11}$ Also, the product was purified by the liquid chromatography methods using medium pressure liquid chromatography (MPLC). The structure of butyryl Cys was characterized by ${ }^{1} \mathrm{H}$ and ${ }^{13} \mathrm{C}$ NMR spectroscopy and MALDI-TOF mass

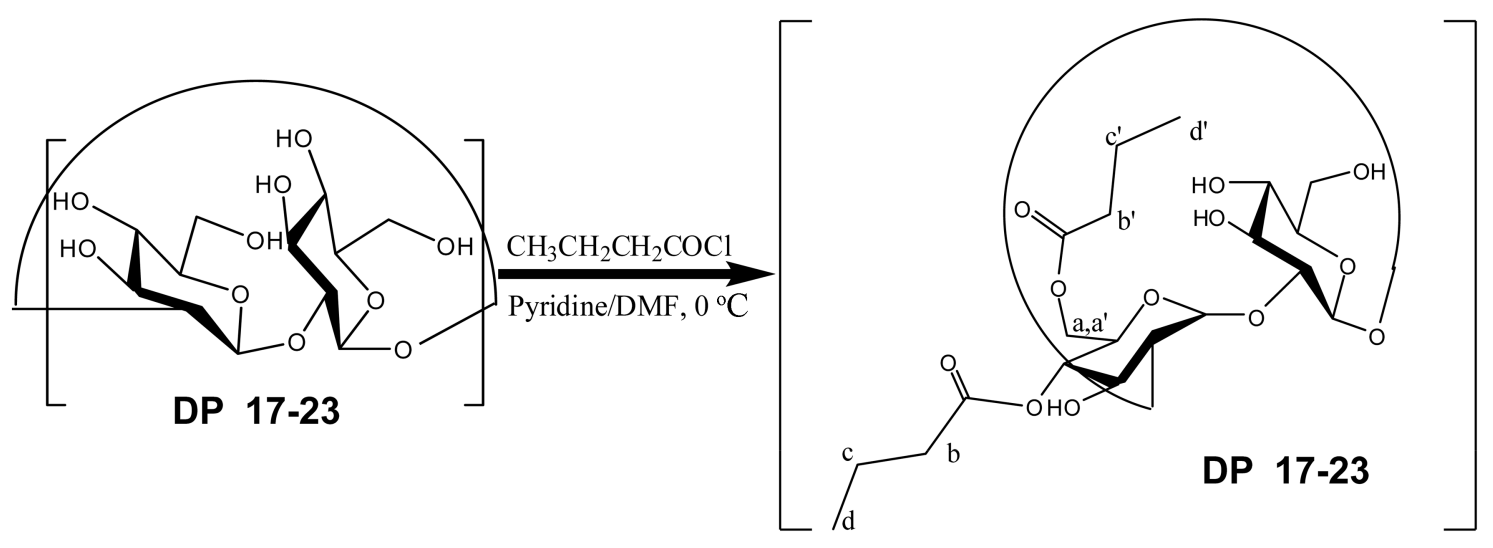

Scheme 1. Butyrylation reaction of Cys. 

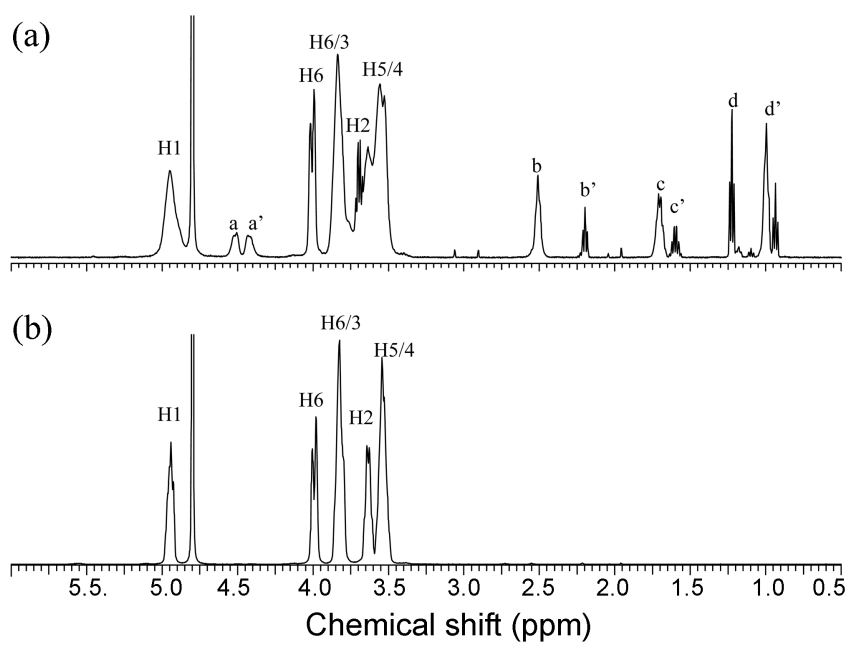

Figure 1. ${ }^{1} \mathrm{H}$ NMR spectra of butyryl Cys (a) and native Cys (b).

spectrometry. In the ${ }^{1} \mathrm{H}$ NMR spectrum, protons of native Cys and butyryl Cys were assigned. Compared with native Cys, some newly detected peaks appeared in butyryl Cys (Figure 1). The peaks between 4.30 and $4.50 \mathrm{ppm}$ are attributed to a and a' of glucose linked with a butyryl residues at C-6 via an ester bond. ${ }^{12}$ The other peaks ranging from 0.94 to $2.51 \mathrm{ppm}$ indicate protons of alkyl chain in the butyryl residue. Peaks from 2.20 to $2.50 \mathrm{ppm}$ (b and $\mathrm{b}^{\prime}$ ) and signals from 1.60 to $1.70 \mathrm{ppm}\left(\mathrm{c}\right.$ and $\mathrm{c}^{\prime}$ ) are assigned to methylene protons $\left(-\mathrm{CH}_{2}-\right)$. Also, the peaks ranging from 0.95 to $1.20\left(\mathrm{~d}\right.$ and $\left.\mathrm{d}^{\prime}\right)$ are attributed to methyl protons $\left(-\mathrm{CH}_{3}\right)$ of the butyryl residues.

In the ${ }^{13} \mathrm{C}$ NMR spectra, the signals around $60.00-110.00$ ppm are assigned to C- 1 to C- 6 carbons of native and butyryl Cys (Figure 2). The butyryl substituents are attached to C-6 and C-4 carbons of the glucose unit, and the substituted C-4' and C-6 carbons are present at 76.32 and $65.61 \mathrm{ppm}$, respectively (Figure 2(a)). Also, peaks at 38.52, 42.35, 20.80 , and $22.09 \mathrm{ppm}$ are attributed to methylene carbons (b, $\mathrm{b}^{\prime}, \mathrm{c}$ and $\mathrm{c}^{\prime}$ ), and signals at 15.99 and $19.55 \mathrm{ppm}$ belong to the methyl carbon ( $\mathrm{d}$ and $\mathrm{d}^{\prime}$ ) of the butyryl residue. The carbonyl carbon $(\mathrm{C}=\mathrm{O})$ of the butanoate residue appears at $179.31 \mathrm{ppm}$. These NMR spectroscopic analyses indicate
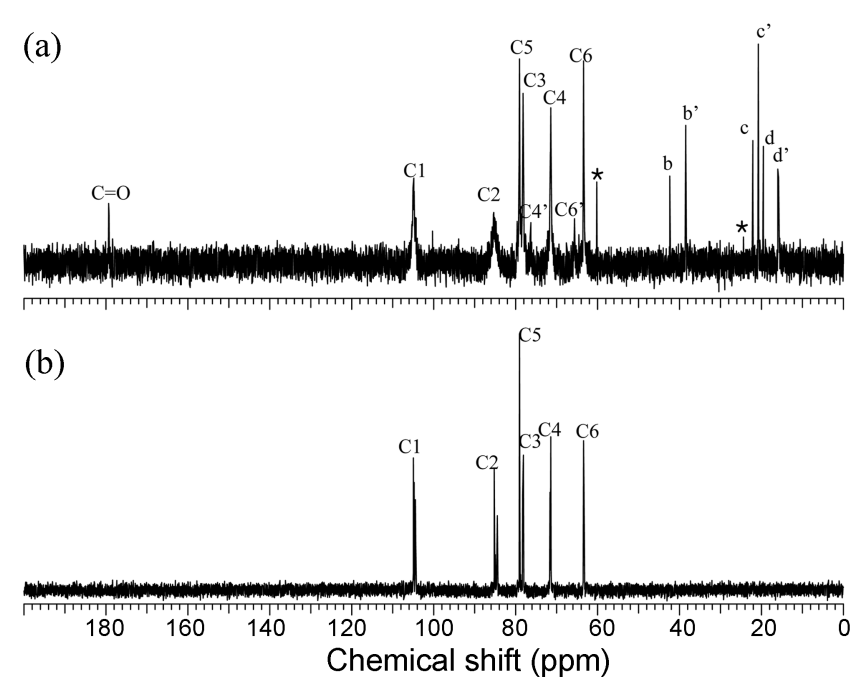

Figure 2. ${ }^{13} \mathrm{C}$ NMR spectra of butyryl Cys (a) and native Cys (b). (*: Impurities came from the residual ethanol.) (a)<smiles>O=c1c(-c2ccc(O)cc2)coc2cc(O)ccc12</smiles><smiles>O=c1c(O)c(-c2ccc(O)cc2)oc2cc(O)cc(O)c12</smiles>

Figure 3. The chemical structures of daidzein (a), genistein (b), kaempferol (c), and myricetin (d).

that the butyryl residues are successfully substituted at the 4th and the 6th positions of native Cys.

To identify the degree of substitution (DS), the butyryl Cys were subjected to MALDI-TOF mass spectrometric analysis. Table 1 shows the measured and calculated mass data of butyryl Cys analyzed by MALDI-TOF mass spectro-

Table 1. Measured and calculated mass units of the $\mathrm{m} / \mathrm{z}$ peaks of butyryl-Cys

\begin{tabular}{|c|c|c|c|c|c|c|c|}
\hline \multirow{2}{*}{$\mathrm{TNS}^{b}$} & \multicolumn{7}{|l|}{$\mathrm{DP}^{a}$} \\
\hline & 17 & 18 & 19 & 20 & 21 & 22 & 23 \\
\hline 1 & $2850(2824)$ & $3011(2986)$ & $3173(3148)$ & $3334(3310)$ & $3497(3472)$ & $3659(3634)$ & \\
\hline 2 & 2919(2894) & $3081(3056)$ & $3243(3218)$ & $3405(3380)$ & $3567(3542)$ & $3729(3704)$ & \\
\hline 3 & 2989(2964) & $3151(3126)$ & $3313(3288)$ & $3475(3450)$ & $3637(3612)$ & $3799(3774)$ & \\
\hline 4 & $3060(3034)$ & $3221(3196)$ & $3383(3358)$ & $3545(3520)$ & $3707(3682)$ & $3869(3844)$ & \\
\hline 5 & $3129(3104)$ & $3292(3266)$ & $3453(3428)$ & $3615(3590)$ & $3777(3752)$ & $3940(3914)$ & \\
\hline 6 & & & & $3685(3660)$ & $3848(3822)$ & & \\
\hline 7 & & & & $3756(3730)$ & $3918(3892)$ & & \\
\hline 8 & & & & $3825(3800)$ & & & \\
\hline 9 & & & & $3895(3870)$ & & & \\
\hline 10 & & & & $3966(3940)$ & & & \\
\hline
\end{tabular}

${ }^{a}$ DP: degree of polymerization. ${ }^{b}$ TNS: a total number of substitution. The numbers in parentheses indicate theoretically calculated values. 
metry in the positive-ion mode. The measured mass units of butyryl Cys indicate the presence of $\left[\mathrm{M}+3 \mathrm{H}^{+}\right],\left[\mathrm{M}+4 \mathrm{H}^{+}\right]$, $\left[\mathrm{M}+\mathrm{Na}^{+}+2 \mathrm{H}^{+}\right]$, and $\left[\mathrm{M}+\mathrm{Na}^{+}+3 \mathrm{H}^{+}\right]$. For example, the highest peak in the mass data appeared at $\mathrm{m} / \mathrm{z} 3383$, indicating [butyryl Cys (DP 19 and DS 4) $+\mathrm{Na}^{+}+2 \mathrm{H}^{+}$]. However, DS value of the butyryl Cys was various depending on the Cys DP range. Cys of DP 17, 18, 19 and 22 are substituted with one to five butyryl residues, and Cys of DP 20 show the highest substitution upto ten butyryl residues. In the case of DP 21, the butyryl residues are substituted with one to seven. Finally, the average DS of butyryl Cys was determined to 5.17 , and the average molecular weight was calculated as 3488.5.

Recently, it has been reported that cyclic glucans and their derivatives as a mixture form have many biotechnological fields based on the host-guest complexation. ${ }^{6,10,13-15}$ In the present study, we investigated butyryl Cys as a novel host molecule for some water-insoluble flavonoids. The complexation ability of butyryl Cys was estimated from its enhancement of the solubilities of poorly soluble flavonoids, using methods I according to Ikeda et al. ${ }^{4}$

Figure 4 shows UV spectra of isoflavones (daidzein and genistein) and flavonols (kaempferol and myricetin) after inclusion complexation with Cys and butyryl Cys. The absorbances of isoflavone in aqueous solution increase in the presence of butyryl Cys, as shown in Figure 4(a) and 4(b). On the other hand, absorbances of flavonols (Figure 4(c) and 4(d)) show no enhancement by butyryl Cys. The differential behavior of the two compounds may be due to the structural difference between isoflavone and flavonol. Actually, isoflavones has the backbone structure different from flavonol, in which ring $\mathrm{B}$ is not attached to the $\mathrm{C}-2$ but to the $\mathrm{C}-3$ position of ring $\mathrm{C}$. In addition, no increase by Cys suggests that the butyryl substituents participate in the isoflavones/ butyryl Cys complexation. Previously, the effect of hydrophobic side chain has been reported with acetyl, propionyl, and butyryl $\beta$-cyclodextrin (CD) complex. ${ }^{10}$ The study has shown that an increase in the hydrophobicity of the substituents enhances the formation of initial complex. In this respect, hydrophobicity of butyryl group may contribute to the isoflavones/butyryl Cys complex formation.

Based on the UV spectra depending on the concentration of butyryl Cys, phase solubility diagrams (see insets of Figure 4(a) and 4(b)) were obtained. According to Higuchi and Connors, ${ }^{16}$ these curves can be classified as $A_{L}$ type, consistent with a 1:1 molecular complex formation. From the slope of the linear fit (correlation coefficient, $\mathrm{R}^{2}=0.990$ and 0.980 ), the stability constants for the $1: 1$ daidzein/ butyryl Cys and genistein/butyryl Cys inclusion complexes were determined to $\mathrm{K}_{\mathrm{s}}=123$, and $83 \mathrm{M}^{-1}$, respectively.
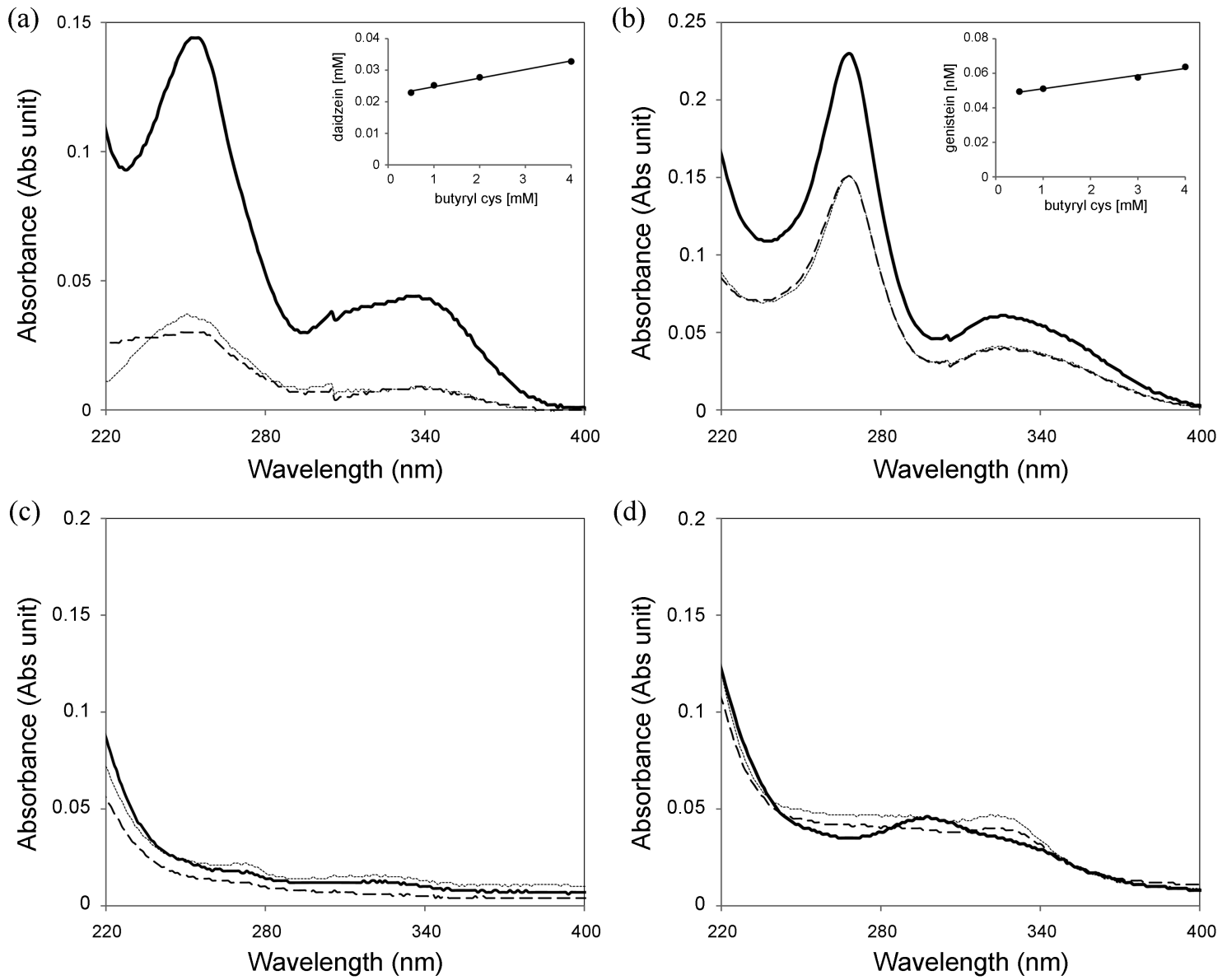

Figure 4. UV-vis adsorption spectra of daidzein (a), genistein (b), kaempferol (c), and myricetin (d) alone in water ( $\cdots)$, with 2 mM Cys (---) and $2 \mathrm{mM}$ butyryl Cys (-). The insets of Figure 4(a) and 4(b) show phase solubility diagrams. 
Daidzein containing less hydroxyl groups showed better affinity with butyryl Cys.

In conclusion, we synthesized butyryl Cys through a onestep chemical modification of Cys and the structure was analyzed by MALDI-TOF mass spectrometry and ${ }^{1} \mathrm{H}$ and ${ }^{13} \mathrm{C}$ NMR spectroscopy. In addition, the butyryl Cys were investigated as a novel host molecule for some insoluble flavonoids. Depending on the backbone structure of flavonoids, the complexation ability of butyryl Cys could be differentiated, and only isoflavones (daidzein and genistein) were complexed with butyryl Cys. By the complexation with butyryl Cys, the solubility of isoflavones was enhanced. In further study, the investigation on effective complexation of butyryl Cys with other hydrophobic guest molecules is expected.

\section{Experimental Section}

Materials. Kaempferol and myricetin were purchased from Indofine chemical company. Daidzein, genistein, pyridine (anhydrous, 99.8\%), DMF ( $N, N$-Dimethylforamide, anhydrous, 99.8\%) and butyryl chloride (99\%) were purchased from Sigma-Aldrich Corp. (St. Louis, MO, USA).

Preparation of Cys. Shinorhizobium leguminosarum Biovar trifolii TA-1 produces Cys. Isolation and purification of Cys were carried out as described in the previous reports. $^{17}$

Preparation of Butyryl Cys. Butyryl Cys were prepared based on the previous report on butyryl CD. ${ }^{10}$ To a stirred solution of $300 \mathrm{mg}$ Cys in $5 \mathrm{~mL}$ of pyridine: DMF (1:1), the required amount of butyryl chloride was added in a dropwise. The reaction mixture was placed in an ice-bath during the reaction time. After the reaction time $(8 \mathrm{hr}), 1 \mathrm{~mL}$ of water was added to terminate the reaction. The solvent was removed by a rotary evaporator, and ethanol was added to the residue. After ethanol addition, the reaction mixture was neutralized to $\mathrm{pH} 7.0$ with $1 \mathrm{~N}$ sodium hydroxide solution. It was then placed in an ice-bath and an adequate amount of ethanol was added to precipitate the product. The product was filtrated and a white powder was obtained. The powder was dried overnight at $70{ }^{\circ} \mathrm{C}$ in vacuo. The dried reaction mixture was separated by MPLC packed with silica gel 60 using mixed solvent of chloroform-ethanol-water $(\mathrm{v} / \mathrm{v} / \mathrm{v}=$ $1: 4: 2$ ) at a flow rate of $2.5 \mathrm{~mL} / \mathrm{min}$. After the separation, the product, butyryl Cys, was obtained and dried.

NMR Spectroscopy and MALDI Mass Spectrometry. The purified Cys and butyryl Cys were confirmed by NMR spectroscopy and MALDI-TOF mass spectrometer. The NMR spectroscopic analyses were carried out on a Bruker AMX spectrometer (operated at $500 \mathrm{MHz}$ for ${ }^{1} \mathrm{H}$ and 125 $\mathrm{MHz}$ for ${ }^{13} \mathrm{C}$ ) at room temperature. The samples were dissolved in deutrated water $\left(\mathrm{D}_{2} \mathrm{O}, 99.96 \%\right)$. All NMR measurents were performed with $0.7 \mathrm{~mL}$ samples in $5 \mathrm{~mm}$ NMR tubes. MALDI-TOF mass spectrometry (VoyagerDETM STR Bio-Spectrometry, Applied Biosystems, Framingham, MA, USA) was carried out in the positive ion mode. In this analysis, 2,5-dihydroxybenzoic acid (DHB) was used as the matrix.
Screening the Flavonoids Solubilized by Complexation of Butyryl Cys. As control, $100 \mu \mathrm{M}$ of flavonol (kaempferol, myricetin) and isoflavone (daidzein, genistein) was added to water. Also, $100 \mu \mathrm{M}$ of above flavonoids was mixed with aqueous solutions of $2 \mathrm{mM}$ Cys and $2 \mathrm{mM}$ butyryl Cys. After equilibrium, the filtered mixture was scanned in the 220-400 nm spectral range with a UV-vis spectrophotometer.

Phase-solubility Study of Isoflavone (daidzein and genistein). A fixed initial amount of daidzein $(100 \mu \mathrm{M})$ and genistein $(200 \mu \mathrm{M})$ exceeding their solubility was added to aqueous solutions of Cys and butyryl Cys (0.0 to $4.0 \mathrm{mM})$, then sonicated $(10 \mathrm{~min})$ in water bath. The mixtures were magnetically stirred for $24 \mathrm{hr}$ at room temperature, shielded from light to prevent any degradation of the molecules. After equilibrium, the suspensions were filtered with $0.2 \mu \mathrm{m}$ filter (PTFE syringe filter, Whatman). The amount of genistein and daidzein dissolved was analyzed with a UV-vis spectrophotometer (UV 2450, Shimadzu Corporation).

The apparent stability constant $\left(\mathrm{K}_{\mathrm{s}}\right)$ of isoflavone/butyryl Cys complex was calculated based on the phase-solubility diagrams according to the following equation:

$$
\mathrm{K}_{\mathrm{s}}\left(\mathrm{M}^{-1}\right)=\text { slope } / \mathrm{S}_{0}(1 \text {-slope })
$$

Acknowledgments. This work was supported by the National Research Foundation of Korea Grant funded by the Korean Government (NRF-2010-0022943 and NRF-2011619-E0002) and by Priority Research Centers Program through the National Research Foundation of Korea (NRF) funded by the Ministry of Education, Science and Technology (2009-0093824). SDG.

\section{References}

1. Abe, M.; Amemura, A.; Higashi, S. Plant Soil 1982, 64, 315.

2. (a) Miller, K. J.; Kennedy, E. P.; Reinhold, V. N. Science 1986, 231, 48. (b) Spaink, H. R. Plant Mol. Biol. 1992, 6, 977.

3. Andre', L.; Mazeau, K.; Taravel, F. R.; Tvaroska I. Int. J. Biol. Macromol. 1995, 17, 189.

4. Koizumi, K.; Okada, Y.; Horiyama, S.; Utamura, T.; Higashiura, T.; Ikeda, M. J. Incl. Phenom. 1984, 2, 891.

5. Lee, S.; Choi, Y.; Lee, S.; Jeong, K.; Jung, S. Chirality 2004, 16, 204.

6. Park, H.; Choi, Y.; Kang, S.; Lee, S.; Kwon, C.; Jung, S. Carbohyd. Polym. 2006, 64, 85.

7. Park, H.; Jung, S. Electrophoresis 2005, 26, 3833.

8. Katiyar, S. K.; Elmets, C. A. Int. J. Oncol. 2001, 18, 1307.

9. Kwon, Y.; Kim, H.; Park, S.; Jung, S. Bull. Korean Chem. Soc. 2010, 31, 3035 .

10. Liu, F.; Kidsig, D. O.; Mitra, A. K. Drug. Dev. Ind. Pharm. 1992, 18,1599 .

11. Still, W. C.; Kahn, M.; Mitra, A. J. Org. Chem. 1978, 43, 2923.

12. Cho, E.; Jeon, Y.; Jung, S. Carbohydr. Res. 2009, 344, 996.

13. Arun, R.; Ashok, K. C. K.; Sravanthi, V. V. N. S. S. Sci. Pharm. 2008, 76, 567.

14. Bettinetti, G.; Mura, P.; Faucci, T. M.; Soeernti, M.; Setti, M. Eur. J. Pharm. Sci. 2002, 15, 21.

15. Martina, K.; Puntambekar, D. S.; Barge, A.; Gallarate, M.; Chirio, D.; Carvotto, G. Carbohydr. Res. 2010, 345, 191.

16. Higuchi, T.; Connors, K. A. Adv. Anal. Chem. Instr. 1965, 4, 117.

17. Breedveld, W. M.; Zevenhuizen, L. P. T. M.; Zehnder, A. J. B. Appl. Environ. Microbiol. 1990, 56, 2080. 